\title{
The Gaussian Multiple Access Diamond
}

\section{Channel}

\author{
Wei Kang Nan Liu Weiwei Chong \\ School of Information Science and Engineering \\ Southeast University, Nanjing, Jiangsu, P. R. China, 210096 \\ wkang@seu.edu.cn_nanliu@seu.edu.cn_chongww@yeah.net
}

\begin{abstract}
In this paper, we study the capacity of the diamond channel. We focus on the special case where the channel between the source node and the two relay nodes are two separate links with finite capacities and the link from the two relay nodes to the destination node is a Gaussian multiple access channel. We call this model the Gaussian multiple access diamond channel. We first propose an upper bound on the capacity. This upper bound is a single-letterization of an $n$-letter upper bound proposed by Traskov and Kramer, and is tighter than the cut-set bound. As for the lower bound, we propose an achievability scheme based on sending correlated codes through the multiple access channel with superposition structure. We then specialize this achievable rate to the Gaussian multiple access diamond channel. Noting the similarity between the upper and lower bounds, we provide sufficient and necessary conditions that a Gaussian multiple access diamond channel has to satisfy such that the proposed upper and lower bounds meet. Thus, for a Gaussian multiple access diamond channel that satisfies these conditions, we have found its capacity.
\end{abstract}

keywords: Correlated codes, diamond channel, Gaussian channel, multiple access channel

This paper was presented in part at IEEE International Symposium on Information Theory (ISIT), 2011. This work is partially supported by the National Basic Research Program of China (973 Program 2012CB316004), the National Natural Science Foundation of China under Grants 61271208, 61201170 and 61221002, the Research Fund of National Mobile Communications Research Laboratory, Southeast University (No. 2014A02), the Project-sponsored by SRF for ROCS, SEM and Qing Lan Project. 


\section{INTRODUCTION}

The diamond channel was first introduced by Schein in 2001 [1]. It models the communication from a source node to a destination node with the help of two relay nodes. The channels between the source node and the two relay nodes form a broadcast channel as the first stage and the channels between the two relay nodes and the destination node form a multiple access channel as the second stage. The capacity of the diamond channel in its general form remains unknown. Achievability results were proposed in [1], while for the general diamond channel, the best known converse result is still the cut-set bound [2]. Capacity has been found for some special classes of discrete diamond channels in [3], [4]. For the Gaussian diamond channel, the capacity is approximated within 1 bit [5]. For the Gaussian $N$-relay diamond channel, a uniform approximation of the capacity has been obtained in [6], where bursty amplify-and-forward was proposed as the achievability scheme and simultaneous optimization over all possible cuts was used for the converse.

The problem of sending correlated codes through a multiple access channel was studied in [7]. This channel model can be regarded as a special class of the diamond channel where the broadcast channel between the source node and the two relay nodes are two separate links of finite capacities. We call this channel model the multiple access diamond channel. Achievability results for the discrete multiple access diamond channel were proposed in [7], [8]. In [8], an uncomputable $n$-letter capacity is also provided which is tighter than the cut-set bound. The capacity is the minimum of four $n$-letter mutual information terms for some joint $n$-letter distribution. Therefore, each of these four $n$-letter terms can be considered as an $n$-letter upper bound on the capacity.

The multiple access diamond channel is related to some other multiple access channel problems, such as sending arbitrarily correlated sources through the multiple access channel [9] (also see [7]), and the multiple access channel with conferencing encoders [10], [11]. The major difference between the multiple access diamond channel and the two multiple access channel problems above is the presence of a centralized encoder in the multiple access diamond channel. This property enables one to construct a pair of correlated codes [7], [8] similar to the achievability of the general broadcast channel by Marton [12].

In this paper, we focus on the multiple access diamond channel where the multiple access 
channel from the two relay nodes to the destination node is Gaussian. We call this channel model the Gaussian multiple access diamond channel. We first obtain an upper bound on the capacity via single-letterization of one of the $n$-letter upper bounds in [8]. The main technique in the upper bound derivation is bounding the correlation between the transmitted signals of the two relays via an auxiliary random variable. This technique was used by Ozarow in solving the Gaussian multiple description problem [13].

As for the lower bound, we first provide an achievable rate for the general, i.e., not necessarily Gaussian, multiple access diamond channel. The achievability scheme we propose is similar to [7], [8], except that the codebook is of a superposition structure. The inner code, which contains part of the message, is decoded by both relay nodes and serves as common data. The outer code, which contains the remaining part of the message, is a pair of correlated codewords and Codeword $k$ is delivered to Relay $k, k=1,2$. The relay nodes send correlated codewords along with the common data into the multiple access channel. This achievable rate is then specialized to the Gaussian multiple access diamond channel.

Finally, we characterize the sufficient and necessary conditions under which the proposed upper bound is strictly tighter than the cut-set bound. Furthermore, noting that the proposed upper and lower bounds take on similar forms, we proceed to provide sufficient and necessary conditions under which our upper and lower bounds meet. Thus, for a Gaussian multiple access diamond channel that satisfies these conditions, we have found its capacity.

The remainder of this paper is organized as follows. In Section II, we provide the system model. In Section III, we derive an upper bound on the capacity of the Gaussian multiple access diamond channel. Achievable schemes and the corresponding rates are described in Section IV. In Section V, we provide conditions under which our upper and lower bounds meet and thus provide the capacity of Gaussian multiple access diamond channels that satisfy these conditions. Proofs are collected in Section VI, which are followed by conclusions in Section VII.

\section{SySTEM MODEL}

Consider a multiple access diamond channel, see Figure 1, The capacity of the link from the source node to Relay $k$ is $C_{k}$, for $k=1,2$. The channel between the two relay nodes and the destination node is a multiple access channel with input alphabets $\left(\mathcal{X}_{1}, \mathcal{X}_{2}\right)$, output alphabet $\mathcal{Y}$ and a transition probability $p\left(y \mid x_{1}, x_{2}\right)$ defined on $\mathcal{Y} \times \mathcal{X}_{1} \times \mathcal{X}_{2}$. Let $W$ be a message that 


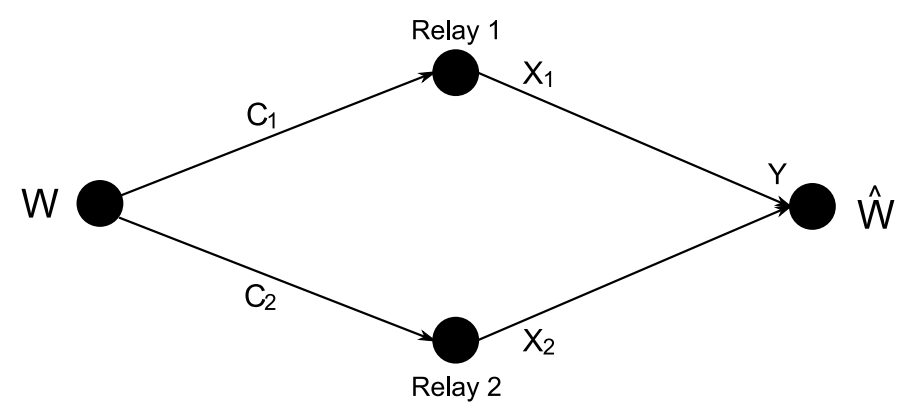

Fig. 1. The multiple access diamond channel.

the source node would like to transmit to the destination node. Assume that $W$ is uniformly distributed on $\{1,2, \cdots, M\}$. An $\left(M, n, \epsilon_{n}\right)$ code consists of an encoding function at the source node

$$
f^{n}:\{1,2, \cdots, M\} \rightarrow\left\{1,2, \cdots, 2^{n C_{1}}\right\} \times\left\{1,2, \cdots, 2^{n C_{2}}\right\}
$$

two encoding functions at the relays

$$
f_{k}^{n}:\left\{1,2, \cdots, 2^{n C_{k}}\right\} \rightarrow \mathcal{X}_{k}^{n}, \quad k=1,2
$$

and a decoding function at the destination node

$$
g^{n}: \mathcal{Y}^{n} \rightarrow\{1,2, \cdots, M\}
$$

The average probability of error is defined as

$$
\epsilon_{n}=\sum_{w=1}^{M} \frac{1}{M} \operatorname{Pr}\left[g^{n}\left(Y^{n}\right) \neq w \mid W=w\right]
$$

Rate $R$ is said to be achievable if there exists a sequence of $\left(2^{n R}, n, \epsilon_{n}\right)$ codes such that $\epsilon_{n} \rightarrow 0$ as $n \rightarrow \infty$. The capacity of the multiple access diamond channel is the supremum of all achievable rates.

In this paper, we focus on the Gaussian multiple access diamond channel, i.e., $\mathcal{X}_{1}=\mathcal{X}_{2}=$ $\mathcal{Y}=\mathbb{R}$ and the channel between the two relay nodes to the destination node is a Gaussian 


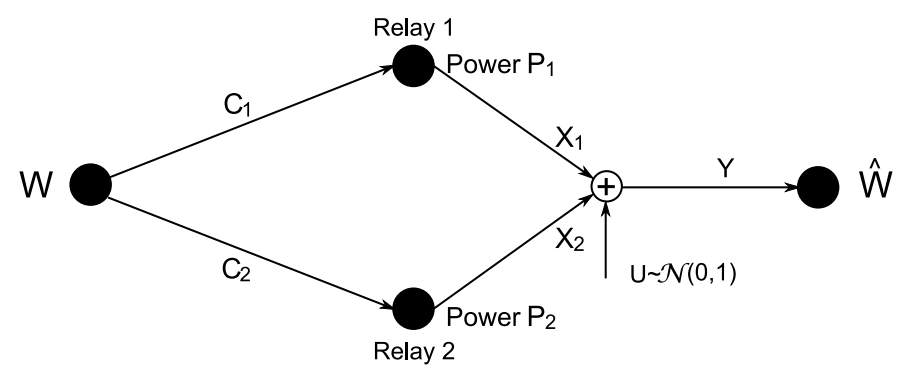

Fig. 2. The Gaussian multiple access diamond channel.

multiple access channel, see Figure 2, The received signal at the destination node is

$$
Y=X_{1}+X_{2}+U
$$

where $X_{1}$ and $X_{2}$ are the input signals from Relay 1 and Relay 2, respectively, and $U$ is a zeromean unit-variance Gaussian random variable. It is assumed that $U$ is independent to $\left(X_{1}, X_{2}\right)$. The encoding functions at the two relay nodes must satisfy the average power constraints: for any codeword $x_{k}^{n}$ that Relay $k$ sends into the Gaussian multiple access channel, it satisfies

$$
\frac{1}{n} \sum_{i=1}^{n} x_{k i}^{2} \leq P_{k}, \quad k=1,2 .
$$

We would like to characterize the capacity of the Gaussian multiple access diamond channel in terms of the channel parameters $C_{1}, C_{2}, P_{1}$ and $P_{2}$.

To simplify presentation, we define the following functions of $\rho$ for $\rho \in[0,1]$ :

$$
\begin{aligned}
& f_{1}(\rho) \triangleq C_{1}+\frac{1}{2} \log \left[1+\left(1-\rho^{2}\right) P_{2}\right], \\
& f_{2}(\rho) \triangleq C_{2}+\frac{1}{2} \log \left[1+\left(1-\rho^{2}\right) P_{1}\right], \\
& f_{3}(\rho) \triangleq C_{1}+C_{2}-\frac{1}{2} \log \left(\frac{1}{1-\rho^{2}}\right), \\
& f_{4}(\rho) \triangleq \frac{1}{2} \log \left(1+P_{1}+P_{2}+2 \rho \sqrt{P_{1} P_{2}}\right) .
\end{aligned}
$$

\section{AN UPPER BOUND}

We first provide an upper bound on the capacity of the Gaussian multiple access diamond channel. 
Theorem 1 An upper bound on the capacity of the Gaussian multiple access diamond channel, denoted as $C_{\text {upper, }}$ is

$$
C_{\text {upper }}=\max \left(T_{1}, T_{2}\right)
$$

where

$$
\begin{aligned}
& T_{1}=\max _{0 \leq \rho \leq \rho^{*}} \min \left\{f_{1}(\rho), f_{2}(\rho), f_{3}(\rho), f_{4}(\rho)\right\}, \\
& T_{2}=\max _{\rho^{*} \leq \rho \leq 1} \min \left\{f_{1}(\rho), f_{2}(\rho), f_{3}(0), f_{4}(\rho)\right\},
\end{aligned}
$$

and

$$
\rho^{*}=\sqrt{1+\frac{1}{4 P_{1} P_{2}}}-\frac{1}{2 \sqrt{P_{1} P_{2}}} .
$$

The proof of Theorem 1 is in Section VI-A

Remark: We note that $T_{1}$ and $T_{2}$ essentially take the same form except the third term which is equal to $f_{3}(\rho)$ for $0 \leq \rho \leq \rho^{*}$ in $T_{1}$ and equal to $f_{3}(0)$ for $\rho^{*} \leq \rho \leq 1$ in $T_{2}$. The cut-set bound for the Gaussian multiple access diamond channel, denoted as $C_{\text {cut }}$, is

$$
C_{\mathrm{cut}}=\max _{0 \leq \rho \leq 1} \min \left\{f_{1}(\rho), f_{2}(\rho), f_{3}(0), f_{4}(\rho)\right\}
$$

Notice that the third term is $f_{3}(0)$ for $0 \leq \rho \leq 1$. Hence, our upper bound is in general tighter than the cut-set bound. Our upper bound is strictly tighter than the cut-set bound when $C_{\text {upper }}$ takes the value of $f_{3}(\rho)$ for some $\rho$ satisfying $0 \leq \rho \leq \rho^{*}$. This will be illustrated analytically in Theorem 3 and also numerically by examples in Section $\mathrm{V}$.

The converse result in Theorem 1 is a single-letterization of one of the $n$-letter upper bounds in [8], which is tighter than the cut-set bound. In the cut-set bound, the cut through the two orthogonal links yields $f_{3}(0)=C_{1}+C_{2}$, which implies that if it is achievable, the signals through the two links should be independent. However, to achieve a larger rate in the second stage, i.e., from the two relays to the destination, the inputs of the multiple access channel should be correlated, in which case $C_{1}+C_{2}$ is no longer achievable. To obtain a tighter bound, it is essential to characterize the correlation between the code pair $X_{1}^{n}$ and $X_{2}^{n}$, i.e., $I\left(X_{1}^{n} ; X_{2}^{n}\right)$, where $X_{k}^{n}$ is the length $n$ sequence transmitted by relay $k, k=1,2$. This is why in the upper bound in [8], $C_{1}+C_{2}$ is replaced by $C_{1}+C_{2}-\frac{1}{n} I\left(X_{1}^{n} ; X_{2}^{n}\right)$. However it is in an $n$-letter form 
and therefore uncomputable.

It is desirable to "single-letterize" the term $I\left(X_{1}^{n} ; X_{2}^{n}\right)$ for a pair of correlated codes, not only in our problem of the multiple access diamond channel, but also in other problems in multi-user information theory. This single-letterization problem remains open in its general form. But for the Gaussian multiple description problem, $I\left(X_{1}^{n} ; X_{2}^{n}\right)$ is single-letterized in [13] by introducing an auxiliary random variable and applying the entropy power inequality [14]. Inspired by [13], we define the auxiliary random variables $Z_{i}=Y_{i}+U_{i}^{\prime}, i=1,2, \cdots, n$, where $Y_{i}$ is the output of the multiple access channel of the $i$-th channel use and $U_{i}^{\prime}$ is a Gaussian random variable with zero mean and variance $N$ and it is independent to everything else. By choosing the right value of $N$, we can obtain a tight upper bound on the term $I\left(X_{1}^{n} ; X_{2}^{n}\right)$. Such non-negative $N$ can be found as long as the correlation between $X_{1}^{n}$ and $X_{2}^{n}$ is not too large. This is the reason why in Theorem 1, the upper bound $f_{3}(\rho)$ can only be enforced for $\rho \leq \rho^{*}$. The details of the proof is provided in Section VI-A

\section{A LOWER BOUND}

In this section, we first provide a lower bound on the capacity of the general, i.e., not necessarily Gaussian, multiple access diamond channel. We then specialize the result to the Gaussian case and obtain an achievable rate for the Gaussian multiple access diamond channel.

Theorem 2 A lower bound on the capacity of the multiple access diamond channel is

$$
\min \left\{\begin{array}{l}
C_{1}+C_{2}-R_{0}-I\left(X_{1} ; X_{2} \mid V\right) \\
C_{1}+I\left(X_{2} ; Y \mid X_{1}, V\right) \\
C_{2}+I\left(X_{1} ; Y \mid X_{2}, V\right) \\
I\left(X_{1}, X_{2} ; Y\right) \\
I\left(X_{1}, X_{2} ; Y \mid V\right)+R_{0}
\end{array}\right\}
$$

for some $0 \leq R_{0} \leq \min \left(C_{1}, C_{2}\right)$ and some joint distribution $p\left(v, x_{1}, x_{2}\right)$.

The details of the proof is provided in Section VI-B Here, we give a brief outline. The achievability scheme we use in obtaining Theorem 2 is the following: the source node uses a codebook of the superposition structure, where $R_{0}$ is the rate of the inner codebook. The source node splits its message into two parts, with rates $R_{0}$ and $R-R_{0}$, respectively. The source node 
encodes the first part of the message into the inner code and delivers the corresponding codeword index to both relays. The source node encodes the second part of the message into the outer code, which consists of a pair of correlated codewords. The index of the first codeword of the pair is sent to Relay 1, while the index of the second codeword of the pair is sent to Relay 2. Each relay finds the codeword from the codebook corresponding to its received indices, and sends the codeword into the multiple access channel. An independent and concurrent version of Theorem 2 is in [15].

From Theorem 2, we obtain an achievable rate for the Gaussian multiple access diamond channel in the following Corollary.

Corollary 1 A lower bound on the capacity of the Gaussian multiple access diamond channel is

$$
\min \left\{\begin{array}{l}
C_{1}+C_{2}-R_{0}-\frac{1}{2} \log \frac{1}{1-\rho^{2}} \\
C_{1}+\frac{1}{2} \log \left[1+\left(1-\rho^{2}\right)\left(1-\beta^{2}\right) P_{2}\right] \\
C_{2}+\frac{1}{2} \log \left[1+\left(1-\rho^{2}\right)\left(1-\alpha^{2}\right) P_{1}\right] \\
\frac{1}{2} \log \left[1+P_{1}+P_{2}+2 \sqrt{P_{1} P_{2}}\left(\alpha \beta+\rho \sqrt{\left(1-\alpha^{2}\right)\left(1-\beta^{2}\right)}\right)\right] \\
\frac{1}{2} \log \left[1+\left(1-\alpha^{2}\right) P_{1}+\left(1-\beta^{2}\right) P_{2}+2 \rho \sqrt{\left(1-\alpha^{2}\right)\left(1-\beta^{2}\right) P_{1} P_{2}}\right]+R_{0}
\end{array}\right\}
$$

for some $0 \leq R_{0} \leq \min \left(C_{1}, C_{2}\right), 0 \leq \alpha, \beta \leq 1$

Proof: Consider the following configuration of $X_{1}, X_{2}$ and $V$ :

$$
\begin{aligned}
& X_{1}=\alpha \sqrt{P_{1}} V+X_{1}^{\prime}, \\
& X_{2}=\beta \sqrt{P_{2}} V+X_{2}^{\prime},
\end{aligned}
$$

where $V \sim \mathcal{N}(0,1)$, and $\left(X_{1}^{\prime}, X_{2}^{\prime}\right)$ are jointly Gaussian with zero mean and covariance matrix

$$
\left[\begin{array}{cc}
\left(1-\alpha^{2}\right) P_{1} & \rho \sqrt{\left(1-\alpha^{2}\right)\left(1-\beta^{2}\right) P_{1} P_{2}} \\
\rho \sqrt{\left(1-\alpha^{2}\right)\left(1-\beta^{2}\right) P_{1} P_{2}} & \left(1-\beta^{2}\right) P_{2}
\end{array}\right] .
$$

Furthermore, $\left(X_{1}^{\prime}, X_{2}^{\prime}\right)$ and $V$ are independent. With this selection of $X_{1}, X_{2}, V$, the achievable rate of (5) follows from Theorem 2 easily.

In Corollary 1, if we set $R_{0}=\alpha=\beta=0$, we obtain a smaller achievable rate: 
Corollary 2 A lower bound of the capacity of the Gaussian multiple access diamond channel, denoted as $C_{\text {lower }}$, is

$$
C_{\text {lower }}=\max _{0 \leq \rho \leq 1} \min \left\{f_{1}(\rho), f_{2}(\rho), f_{3}(\rho), f_{4}(\rho)\right\}
$$

Remark: The achievable rate in Corollary 2 is in general smaller than that in Corollary 1. More specifically, when $C_{1}$ and $C_{2}$ are sufficiently large, the multiple access channel in the second stage is the bottleneck of the network, and the optimal achievable scheme is sending fully correlated codewords into the multiple access channel, resulting in the achievable rate of $f_{4}(1)$. But the correlated codes without the superposition structure, used in Corollary 2, can not

support fully correlated codewords, because $f_{4}(1)>f_{3}(1)$, which means $C_{\text {lower }}<f_{4}(1)$. This shows that the superposition scheme in Corollary 1 strictly outperforms the scheme without superposition structure in the case with large $C_{1}$ and $C_{2}$.

Though the lower bound in Corollary 2 is smaller than that of Corollary 1, it takes on a similar form as the upper bound in Theorem 1. Thus, when the channel satisfy certain conditions, we expect the upper bound in Theorem 1 and the lower bound in Corollary 2 to coincide, yielding the capacity. This will be discussed in the next section.

\section{CAPACITY}

Comparing the upper and lower bounds proposed in Theorem 1 and Corollary 2, we see that they take on similar forms. More specifically, the four functions after the minimum in (2) are exactly the same as that in (6). Thus, it can be expected that for certain parameters of the Gaussian multiple access diamond channel, $C_{1}, C_{2}, P_{1}$ and $P_{2}$, the upper and lower bounds meet providing us with the exact capacity of the channel. We now proceed to give explicit conditions on the channel parameters such that our upper and lower bounds meet.

First, we eliminate the trivial cases. If the channel is such that

$$
\min \left(C_{1}, C_{2}\right) \geq \frac{1}{2} \log \left(1+P_{1}+P_{2}+2 \sqrt{P_{1} P_{2}}\right)
$$

then the multiple access channel in the second stage is the bottleneck of the whole network, and thus, the capacity of the Gaussian multiple access diamond channel is equal to

$$
\frac{1}{2} \log \left(1+P_{1}+P_{2}+2 \sqrt{P_{1} P_{2}}\right) .
$$


On the other hand, if the channel is such that

$$
\min \left(C_{1}+\frac{1}{2} \log \left(1+P_{2}\right), C_{2}+\frac{1}{2} \log \left(1+P_{1}\right), C_{1}+C_{2}\right) \leq \frac{1}{2} \log \left(1+P_{1}+P_{2}\right)
$$

then the two separate links in the first stage, or one of the two cross-cuts, i.e., Cut 1 or Cut 2 in Figure 3, is the bottleneck of the whole network, and the capacity is equal to

$$
\min \left(C_{1}+\frac{1}{2} \log \left(1+P_{2}\right), C_{2}+\frac{1}{2} \log \left(1+P_{1}\right), C_{1}+C_{2}\right) .
$$

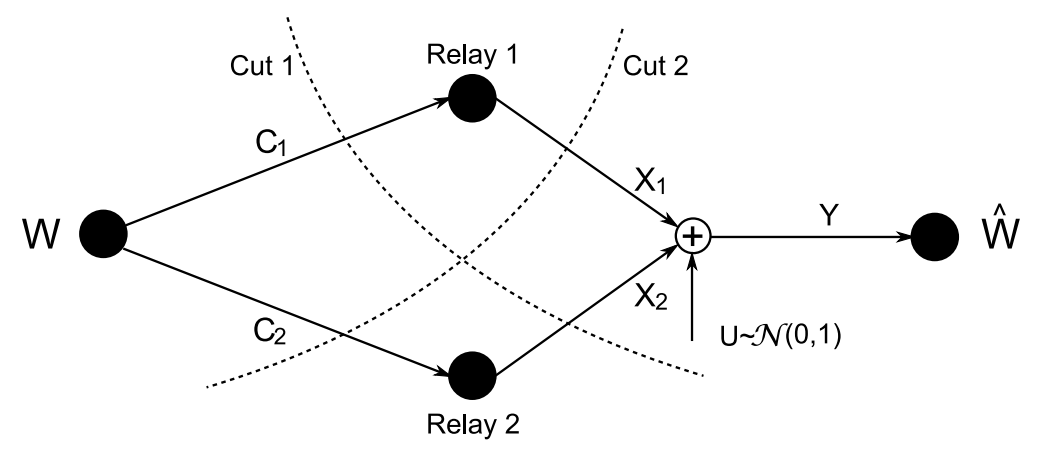

Fig. 3. The cross-cuts of the diamond channel.

Thus, we only need to focus on the nontrivial cases where

$$
\begin{aligned}
\min \left(C_{1}, C_{2}\right) & <\frac{1}{2} \log \left(1+P_{1}+P_{2}+2 \sqrt{P_{1} P_{2}}\right), \text { and } \\
\frac{1}{2} \log \left(1+P_{1}+P_{2}\right) & <\min \left(C_{1}+\frac{1}{2} \log \left(1+P_{2}\right), C_{2}+\frac{1}{2} \log \left(1+P_{1}\right), C_{1}+C_{2}\right)
\end{aligned}
$$

are both satisfied. In the next theorem, we provide sufficient and necessary conditions on the channel parameters for the nontrivial cases described in (7) and (8), such that the upper and lower bounds proposed in Theorem 1 and Corollary 2 meet, yielding the exact capacity, and also the conditions, under which the cut-set bound is strictly larger than the proposed upper bound.

Theorem 3 Consider a Gaussian multiple access diamond channel that satisfies (7) and (8). Define $f_{5}(\rho)=\min \left(f_{1}(\rho), f_{2}(\rho)\right), \forall \rho \in[0,1]$. Let $\bar{\rho}_{k}$ denote the positive root of the equation $f_{k}(\rho)=f_{4}(\rho)$ that is in $[0,1], k=3,5$. Recall that $\rho^{*}$ is defined in Theorem 1. 
1) The sufficient and necessary condition for $C_{\text {lower }}=C_{\text {upper }}$ to hold is

$$
\bar{\rho}_{5} \leq \bar{\rho}_{3} \quad \text { or } \quad f_{4}\left(\bar{\rho}_{3}\right) \geq f_{5}\left(\rho^{*}\right),
$$

and in this case, the capacity is $f_{4}\left(\bar{\rho}_{3}\right)$.

2) The sufficient and necessary condition for $C_{\text {upper }}<C_{\text {cut }}$ to hold is

$$
\bar{\rho}_{3}<\bar{\rho}_{5} \quad \text { and } \quad \rho^{*} \geq \bar{\rho}_{5} \quad \text { and } \quad f_{3}(0)>f_{5}\left(\rho^{*}\right)
$$

3) The sufficient and necessary condition for $C_{\text {lower }}=C_{\text {upper }}<C_{\text {cut }}$ to hold is

$$
\bar{\rho}_{3}<\bar{\rho}_{5} \quad \text { and } \quad f_{4}\left(\bar{\rho}_{3}\right) \geq f_{5}\left(\rho^{*}\right)
$$

and in this case, the capacity is $f_{4}\left(\bar{\rho}_{3}\right)$.

The proof of Theorem 3 is provided in Section VI-C, Theorem 3 illustrates the tightness of the novel upper bound on capacity proposed in Theorem 1, in that for some channel parameters, it is achievable and strictly smaller than the cut-set bound. To the best of our knowledge, Theorem 3 demonstrates the first time where the capacity of certain Gaussian multiple access diamond channels has been characterized when the cut-set bound is not tight.

To show that there indeed exist Gaussian multiple access diamond channels that satisfy the condition (10) stated in Theorem 3, we give the following examples:

1) $C_{1}=1.8, C_{2}=2, P_{1}=10, P_{2}=15$, which means $\rho^{*}=0.9600, \bar{\rho}_{3}=0.8696, \bar{\rho}_{5}=$ $0.8947, f_{4}\left(\bar{\rho}_{3}\right)=2.7819, f_{5}\left(\rho^{*}\right)=2.3608$. Thus, (10) is satisfied and the capacity is $f_{4}\left(\bar{\rho}_{3}\right)=2.7819$ bits/channel use.

2) $C_{1}=2, C_{2}=1.2, P_{1}=30, P_{2}=20$, which means $\rho^{*}=0.9798, \bar{\rho}_{3}=0.4029, \bar{\rho}_{5}=$ $0.7027, f_{4}\left(\bar{\rho}_{3}\right)=3.0722, f_{5}\left(\rho^{*}\right)=1.7688$. Thus, (10) is satisfied and the capacity is $f_{4}\left(\bar{\rho}_{3}\right)=3.0722$ bits/channel use.

Since the conditions provided in Theorem 3 is rather involved, we provide some intuition as to when our proposed upper bound in Theorem 1 and the lower bound in Corollary 2 meet. As noted after the statement of Theorem 1, the upper bound $f_{3}(\rho)$ can only be enforced for $\rho \leq \rho^{*}$. We also observe that $\rho^{*}$ monotonically increases with respect to $P_{1}$ and $P_{2}$. Therefore, the proposed upper bound is tight with large $P_{1}$ and/or $P_{2}$. From the perspective of achievability, as discussed in the remark after Corollary 2, when $C_{1}$ and $C_{2}$ are large, the superposition scheme 


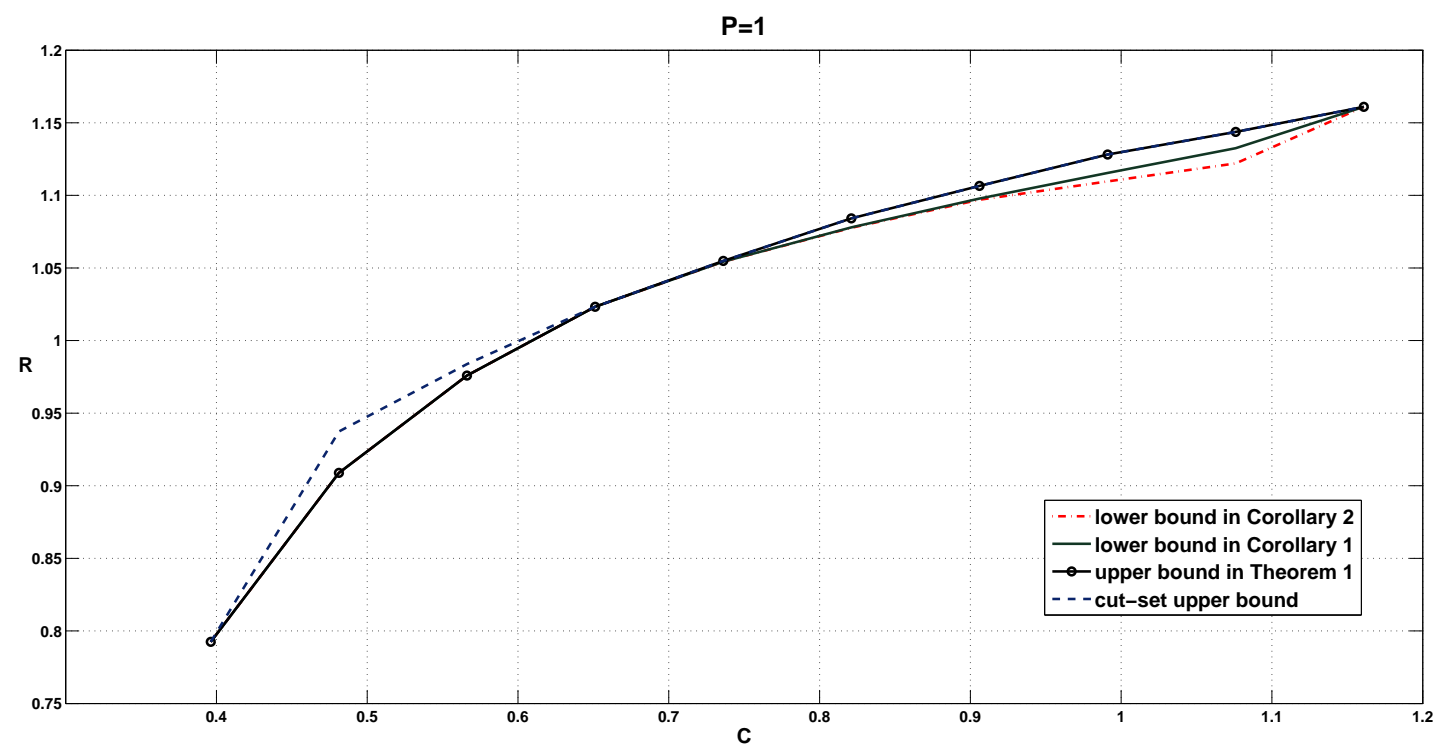

Fig. 4. Comparison of upper and lower bounds with $P=1$.

in Corollary 1 outperforms the scheme without the superposition structure in Corollary 2 i.e., the rate characterized by Corollary 2 is not tight in this case. From the above discussions, we can see that the proposed upper bound in Theorem 1 and the lower bound in Corollary 21 meet when $P_{1}$ and $P_{2}$ are large and/or $C_{1}$ and $C_{2}$ are small. This is also supported by our numerical results discussed next.

We plot the upper and lower bounds in Theorem 1, Corollary 1 and Corollary 2 and depict them in Figures 4 and 5 for the symmetric cases of $C_{1}=C_{2} \triangleq C, P_{1}=P_{2} \triangleq P=1$ and $C_{1}=C_{2} \triangleq C, P_{1}=P_{2} \triangleq P=10$, respectively. The cut-set bound is also plotted to show the improvement of our upper bound over the cut set bound, which was the best known upper bound on the capacity. As can be seen, for small $C$, the proposed upper bound is strictly smaller than the cut-set bound. Also, the gap between our lower and upper bounds is rather small, especially when $C$ is relatively small and/or $P$ is relatively large.

\section{Proofs}

\section{A. Proof of Theorem [1]}

In order to make the proof easier to follow, we split the entire proof into 4 steps, consisting of Lemmas 1 to 4 . 


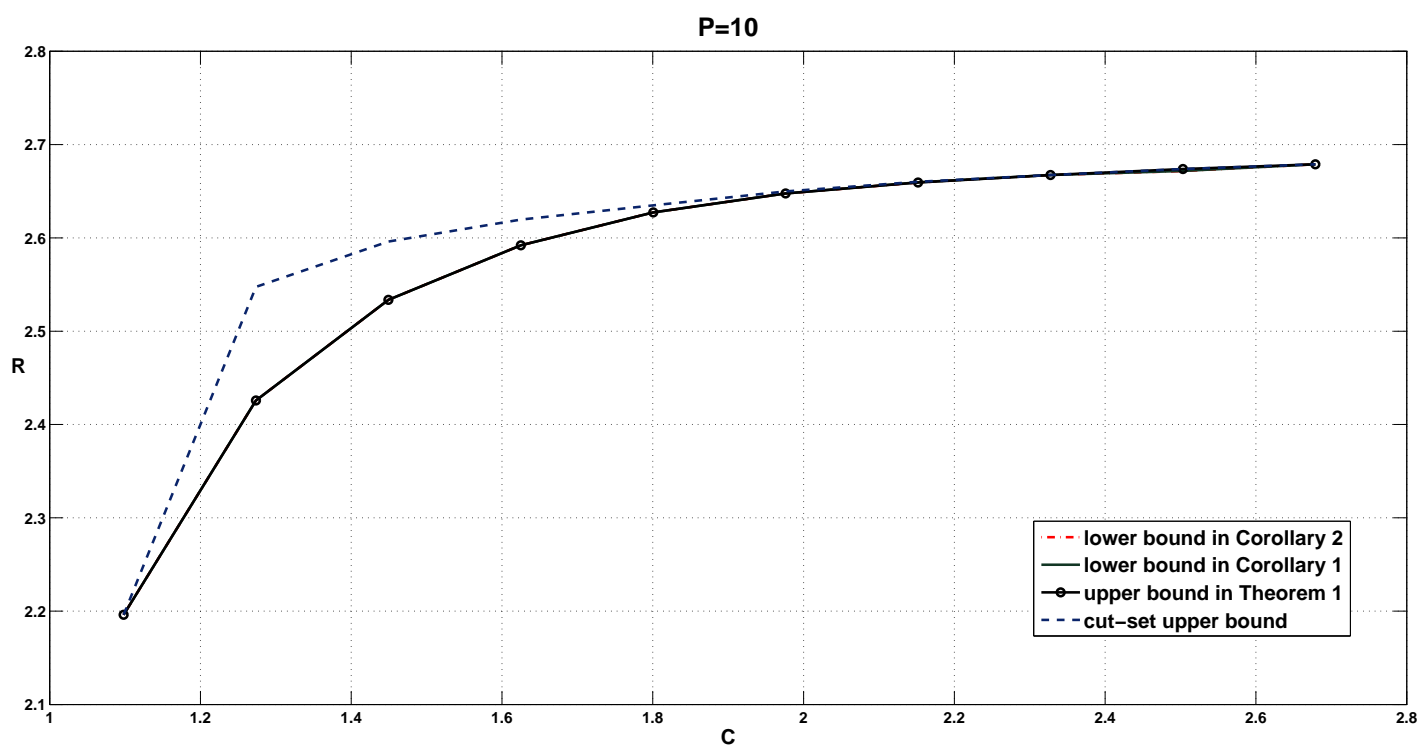

Fig. 5. Comparison of upper and lower bounds with $P=10$.

Starting from Fano's inequality, any achievable rate $R$ must satisfy

$$
\begin{aligned}
n R & =H(W) \\
& =I\left(W ; Y^{n}\right)+H\left(W \mid Y^{n}\right) \\
& \leq I\left(X_{1}^{n}, X_{2}^{n} ; Y^{n}\right)+H\left(W \mid Y^{n}\right) \\
& \leq I\left(X_{1}^{n}, X_{2}^{n} ; Y^{n}\right)+n \epsilon_{n} .
\end{aligned}
$$

where (11) is because of the Markov chain $W \rightarrow\left(X_{1}^{n}, X_{2}^{n}\right) \rightarrow Y^{n}$.

Towards proving that $R \leq \min \left(f_{1}(\rho), f_{2}(\rho)\right)$ for some $\rho \in[0,1]$, we first show the following Lemma.

Lemma 1 There exists $\rho_{a} \in[0,1]$ and $\rho_{b} \in[0,1]$ such that

$$
\begin{aligned}
& R \leq \frac{1}{2} \log \left[\left(1-\rho_{a}^{2}\right) P_{1}+1\right]+C_{2}+\epsilon_{n}, \\
& R \leq \frac{1}{2} \log \left[\left(1-\rho_{b}^{2}\right) P_{2}+1\right]+C_{1}+\epsilon_{n} .
\end{aligned}
$$


Proof: Following from (12), we have

$$
\begin{aligned}
n R & \leq I\left(X_{1}^{n}, X_{2}^{n} ; Y^{n}\right)+n \epsilon_{n} \\
& =I\left(X_{1}^{n} ; Y^{n} \mid X_{2}^{n}\right)+I\left(X_{2}^{n} ; Y^{n}\right)+n \epsilon_{n} \\
& \leq I\left(X_{1}^{n} ; Y^{n} \mid X_{2}^{n}\right)+H\left(X_{2}^{n}\right)+n \epsilon_{n} \\
& \leq I\left(X_{1}^{n} ; Y^{n} \mid X_{2}^{n}\right)+n C_{2}+n \epsilon_{n} \\
& \leq \sum_{i=1}^{n} I\left(X_{1 i} ; Y_{i} \mid X_{2 i}\right)+n C_{2}+n \epsilon_{n} \\
& \leq \sum_{i=1}^{n} \frac{1}{2} \log \left[\left(1-\rho_{i}^{2}\right) P_{1 i}+1\right]+n C_{2}+n \epsilon_{n}
\end{aligned}
$$

(16) is because without loss of generality, we only consider deterministic encoders, thus from (1) we have $H\left(X_{k}^{n}\right) \leq n C_{k}, k=1,2$; (17) follows from the memoryless nature of the channel $p\left(y \mid x_{1}, x_{2}\right) ;(18)$ follows because we have defined $P_{k i} \triangleq E\left[X_{k i}^{2}\right], k=1,2$ and $\rho_{i}=\frac{E\left[X_{1 i} X_{2 i}\right]}{\sqrt{P_{1 i} P_{2 i}}}$, and used the fact that given power constraint, the Gaussian distribution maximizes the differential entropy [2].

Since the inputs from Relay 1 must satisfy the average power constraint $P_{1}$, we have

$$
0 \leq \frac{1}{n} \sum_{i=1}^{n} \rho_{i}^{2} P_{1 i} \leq \frac{1}{n} \sum_{i=1}^{n} P_{1 i} \leq P_{1} .
$$

Therefore, there exists a $\rho_{a} \in[0,1]$ such that

$$
\rho_{a}^{2} P_{1}=\frac{1}{n} \sum_{i=1}^{n} \rho_{i}^{2} P_{1 i} .
$$

Due to the concavity of the logarithm function, we have

$$
\begin{aligned}
R & \leq \frac{1}{n} \sum_{i=1}^{n} \frac{1}{2} \log \left[\left(1-\rho_{i}^{2}\right) P_{1 i}+1\right]+C_{2}+\epsilon_{n} \\
& \leq \frac{1}{2} \log \left(\frac{1}{n} \sum_{i=1}^{n}\left[\left(1-\rho_{i}^{2}\right) P_{1 i}+1\right]\right)+C_{2}+\epsilon_{n} \\
& =\frac{1}{2} \log \left(\frac{1}{n} \sum_{i=1}^{n} P_{1 i}-\frac{1}{n} \sum_{i=1}^{n} \rho_{i}^{2} P_{1 i}+1\right)+C_{2}+\epsilon_{n} \\
& \leq \frac{1}{2} \log \left[\left(1-\rho_{a}^{2}\right) P_{1}+1\right]+C_{2}+\epsilon_{n} .
\end{aligned}
$$


Similarly, there exists a $\rho_{b} \in[0,1]$ such that

$$
\rho_{b}^{2} P_{2}=\frac{1}{n} \sum_{i=1}^{n} \rho_{i}^{2} P_{2 i}
$$

and we have

$$
R \leq \frac{1}{2} \log \left[\left(1-\rho_{b}^{2}\right) P_{2}+1\right]+C_{1}+\epsilon_{n} .
$$

This completes the proof of Lemma 1 .

The following lemma shows the relationship between the achievable rate $R$ and the n-letter entropy $h\left(Y^{n}\right)$.

\section{Lemma 2}

$$
h\left(Y^{n}\right)-\frac{n}{2} \log (2 \pi e) \leq n R \leq h\left(Y^{n}\right)-\frac{n}{2} \log (2 \pi e)+n \epsilon_{n} .
$$

Proof: From (12), we have

$$
\begin{aligned}
n R & \leq h\left(Y^{n}\right)-h\left(Y^{n} \mid X_{1}^{n}, X_{2}^{n}\right)+n \epsilon_{n} \\
& =h\left(Y^{n}\right)-\frac{n}{2} \log (2 \pi e)+n \epsilon_{n} .
\end{aligned}
$$

On the other hand, we also have

$$
\begin{aligned}
n R & =H(W) \\
& \geq H\left(X_{1}^{n}, X_{2}^{n}\right) \\
& \geq I\left(X_{1}^{n}, X_{2}^{n} ; Y^{n}\right) \\
& =h\left(Y^{n}\right)-\frac{n}{2} \log (2 \pi e),
\end{aligned}
$$

where (21) is because $\left(X_{1}^{n}, X_{2}^{n}\right)$ is a deterministic function of $W$. This completes the proof of Lemma 2 .

From Lemma 2, we see that to characterize the achievable rate $R$, we need to characterize $h\left(Y^{n}\right)$. Towards this end, let us define $\rho \in[0,1]$, which is a function of $h\left(Y^{n}\right)$ as follows: If

$$
\frac{1}{n} h\left(Y^{n}\right) \leq \frac{1}{2} \log (2 \pi e)\left(1+P_{1}+P_{2}\right)
$$


then $\rho=0$; otherwise, $\rho$ is such that

$$
\frac{1}{n} h\left(Y^{n}\right)=\frac{1}{2} \log (2 \pi e)\left(1+P_{1}+P_{2}+2 \rho \sqrt{P_{1} P_{2}}\right) .
$$

Lemma $3 \rho$ as defined above satisfies $\rho \leq \min \left(\rho_{a}, \rho_{b}\right)$.

Proof: We upper bound $h\left(Y^{n}\right)$ as

$$
\begin{aligned}
\frac{1}{n} h\left(Y^{n}\right) & \leq \frac{1}{n} \sum_{i=1}^{n} h\left(Y_{i}\right) \\
& \leq \frac{1}{n} \sum_{i=1}^{n} \frac{1}{2} \log (2 \pi e)\left(P_{1 i}+P_{2 i}+2 \rho_{i} \sqrt{P_{1 i} P_{2 i}}+1\right) \\
& \leq \frac{1}{2} \log (2 \pi e)\left(\frac{1}{n} \sum_{i=1}^{n}\left[P_{1 i}+P_{2 i}+2\left|\rho_{i}\right| \sqrt{P_{1 i} P_{2 i}}+1\right]\right) \\
& \leq \frac{1}{2} \log (2 \pi e)\left(P_{1}+P_{2}+\frac{1}{n} \sum_{i=1}^{n} 2 \sqrt{\rho_{i}^{2} P_{1 i} P_{2 i}}+1\right)
\end{aligned}
$$

where (25) follows from the same reason as (18); (26) follows from the concavity of the logarithmic function; (27) follows from the same argument as (19). From Cauchy-Schwarz inequality, we have

$$
\frac{1}{n} \sum_{i=1}^{n} \sqrt{\rho_{i}^{2} P_{1 i} P_{2 i}} \leq \sqrt{\left(\frac{1}{n} \sum_{i=1}^{n} \rho_{i}^{2} P_{1 i}\right)\left(\frac{1}{n} \sum_{i=1}^{n} P_{2 i}\right)} \leq \sqrt{\rho_{a}^{2} P_{1} P_{2}} .
$$

Thus, we have

$$
\frac{1}{n} h\left(Y^{n}\right) \leq \frac{1}{2} \log (2 \pi e)\left(P_{1}+P_{2}+2 \rho_{a} \sqrt{P_{1} P_{2}}+1\right) .
$$

By symmetry, we also have

$$
\frac{1}{n} h\left(Y^{n}\right) \leq \frac{1}{2} \log (2 \pi e)\left(P_{1}+P_{2}+2 \rho_{b} \sqrt{P_{1} P_{2}}+1\right)
$$

From (28), (29) and the definition of $\rho$, we have that $\rho \leq \min \left(\rho_{a}, \rho_{b}\right)$ which completes the proof of Lemma 3 .

As $n \rightarrow \infty$, Lemma 1 together with Lemma 3 means that $R \leq \min \left(f_{1}(\rho), f_{2}(\rho)\right)$, where $\rho$ is defined right before Lemma 3 . Lemma 2 together with the definition of $\rho$ means that $R \leq f_{4}(\rho)$. Thus, we have shown that $R \leq \min \left(f_{1}(\rho), f_{2}(\rho), f_{4}(\rho)\right)$ and it remains to show that if $\rho$ further 
satisfies $0 \leq \rho \leq \rho^{*}$, we have $R \leq f_{3}(\rho)$.

If $\rho=0$, from the cut-set bound, we have $R \leq C_{1}+C_{2}=f_{3}(0)$. As for the case of $0<\rho \leq \rho^{*}$, using Ozarow's idea in [13], we have the following lemma.

Lemma 4 If $\rho$ further satisfies $0<\rho \leq \rho^{*}$, we have

$$
2 R \leq \frac{1}{2} \log \left(1+P_{1}+P_{2}+2 \rho \sqrt{P_{1} P_{2}}\right)+C_{1}+C_{2}-\frac{1}{2} \log \left(\frac{1}{1-\rho^{2}}\right)+2 \epsilon_{n} .
$$

Proof: If $\rho$ satisfies $0<\rho \leq \rho^{*}$, which is equivalent to $\sqrt{P_{1} P_{2}}\left(\frac{1}{\rho}-\rho\right)-1 \geq 0$, we define additional random variables

$$
Z_{i}=Y_{i}+U_{i}^{\prime}, \quad i=1, \ldots, n,
$$

where $U^{\prime n}$ is an i.i.d. Gaussian sequence with mean zero and variance

$$
N=\sqrt{P_{1} P_{2}}\left(\frac{1}{\rho}-\rho\right)-1
$$

and is independent to everything else. We have

$$
\begin{aligned}
2 n R & \leq 2 I\left(X_{1}^{n}, X_{2}^{n} ; Y^{n}\right)+2 n \epsilon_{n} \\
& \leq I\left(X_{1}^{n}, X_{2}^{n} ; Y^{n}\right)+H\left(X_{1}^{n}, X_{2}^{n}\right)+2 n \epsilon_{n} \\
& =I\left(X_{1}^{n}, X_{2}^{n} ; Y^{n}\right)+H\left(X_{1}^{n}\right)+H\left(X_{2}^{n}\right)-I\left(X_{1}^{n} ; X_{2}^{n}\right)+2 n \epsilon_{n} \\
& \leq I\left(X_{1}^{n}, X_{2}^{n} ; Y^{n}\right)+n C_{1}+n C_{2}-I\left(X_{1}^{n} ; X_{2}^{n}\right)+2 n \epsilon_{n},
\end{aligned}
$$

where (32) is the same as (12), and (33) follows from the same reasoning as (16). Note that

$$
\begin{aligned}
I\left(X_{1}^{n} ; X_{2}^{n}\right) & =I\left(X_{1}^{n} ; Z^{n}\right)-I\left(X_{1}^{n} ; Z^{n} \mid X_{2}^{n}\right)+I\left(X_{1}^{n} ; X_{2}^{n} \mid Z^{n}\right) \\
& \geq I\left(X_{1}^{n} ; Z^{n}\right)-I\left(X_{1}^{n} ; Z^{n} \mid X_{2}^{n}\right) \\
& =I\left(X_{1}^{n}, X_{2}^{n} ; Z^{n}\right)-I\left(X_{2}^{n} ; Z^{n} \mid X_{1}^{n}\right)-I\left(X_{1}^{n} ; Z^{n} \mid X_{2}^{n}\right) .
\end{aligned}
$$


We further have

$$
\begin{aligned}
I\left(X_{1}^{n} ; Z^{n} \mid X_{2}^{n}\right) & \leq \sum_{i=1}^{n} \frac{1}{2} \log \frac{\left(1-\rho_{i}^{2}\right) P_{1 i}+1+N}{1+N} \\
& \leq \frac{n}{2} \log \frac{\left(1-\rho^{2}\right) P_{1}+1+N}{1+N},
\end{aligned}
$$

where (35) follows by similar arguments as (18), and (36) follows by using similar arguments as (19) and the result of Lemma 3. Similarly, we have

$$
I\left(X_{2}^{n} ; Z^{n} \mid X_{1}^{n}\right) \leq \frac{n}{2} \log \frac{\left(1-\rho^{2}\right) P_{2}+1+N}{1+N} .
$$

We also have

$$
\begin{aligned}
I\left(X_{1}^{n}, X_{2}^{n} ; Z^{n}\right) & =h\left(Z^{n}\right)-h\left(Z^{n} \mid X_{1}^{n}, X_{2}^{n}\right) \\
& =h\left(Z^{n}\right)-\sum_{i=1}^{n} \frac{1}{2} \log (2 \pi e)(1+N) .
\end{aligned}
$$

From entropy power inequality (EPI) [14, Lemma I], we have

$$
h\left(Z^{n}\right) \geq \frac{n}{2} \log \left[2^{\left(\frac{2}{n} h\left(Y^{n}\right)\right)}+2 \pi e N\right] .
$$

Therefore,

$$
\begin{aligned}
h\left(Z^{n}\right)-h\left(Y^{n}\right) & \geq \frac{n}{2} \log \left[1+\frac{2 \pi e N}{2^{\left(\frac{2}{n} h\left(Y^{n}\right)\right)}}\right] \\
& =\frac{n}{2} \log \left[1+\frac{N}{P_{1}+P_{2}+2 \rho \sqrt{P_{1} P_{2}}+1}\right] \\
& =\frac{n}{2} \log \frac{P_{1}+P_{2}+2 \rho \sqrt{P_{1} P_{2}}+1+N}{P_{1}+P_{2}+2 \rho \sqrt{P_{1} P_{2}}+1}
\end{aligned}
$$

where (38) follows from (24). Thus,

$$
I\left(X_{1}^{n}, X_{2}^{n} ; Y^{n}\right)-I\left(X_{1}^{n}, X_{2}^{n} ; Z^{n}\right) \leq \frac{n}{2} \log \frac{(N+1)\left(P_{1}+P_{2}+2 \rho \sqrt{P_{1} P_{2}}+1\right)}{P_{1}+P_{2}+2 \rho \sqrt{P_{1} P_{2}}+1+N} .
$$


Using (33), (34), (36), (37) and (39), we have

$$
\begin{aligned}
2 n R \leq & \frac{n}{2} \log \left(P_{1}+P_{2}+2 \rho \sqrt{P_{1} P_{2}}+1\right)+n C_{1}+n C_{2} \\
& -\frac{n}{2} \log \frac{\left(P_{1}+P_{2}+2 \rho \sqrt{P_{1} P_{2}}+1+N\right)(1+N)}{\left(\left(1-\rho^{2}\right) P_{1}+1+N\right)\left(\left(1-\rho^{2}\right) P_{2}+1+N\right)}+2 n \epsilon_{n} .
\end{aligned}
$$

Plugging in $N$ defined in (31), we have proved (30), which completes the proof of Lemma 4.

Hence, for the case of $0 \leq \rho \leq \rho^{*}$, letting $n \rightarrow \infty$, from Lemma 2 and Lemma 4, we have proved $R \leq f_{3}(\rho)$. As for the case where $\rho^{*}<\rho \leq 1$, though the result of Lemma 4 no longer holds, from the cut-set bound, we always have $R \leq C_{1}+C_{2}$, which means $R \leq f_{3}(0)$.

For all cases of $\rho \in[0,1]$, we have proved that the achievable rate satisfies either

$$
R \leq \max _{0 \leq \rho \leq \rho^{*}} \min \left\{f_{1}(\rho), f_{2}(\rho), f_{3}(\rho), f_{4}(\rho)\right\}
$$

or

$$
R \leq \max _{\rho^{*} \leq \rho \leq 1} \min \left\{f_{1}(\rho), f_{2}(\rho), f_{3}(0), f_{4}(\rho)\right\}
$$

and thus, Theorem 1 is proved. 
B. Proof of Theorem 2

For a given distribution $p\left(v, x_{1}, x_{2}\right)$, consider a rate tuple $\left(R_{0}, R_{1}, R_{2}, r_{1}, r_{2}\right)$ such that

$$
\begin{aligned}
& r_{1}+r_{2} \geq I\left(X_{1} ; X_{2} \mid V\right)+\delta, \\
& R_{1} \leq I\left(X_{1} ; Y, X_{2} \mid V\right), \\
& R_{2} \leq I\left(X_{2} ; Y, X_{1} \mid V\right), \\
& R_{1}+ R_{2} \leq I\left(X_{1}, X_{2} ; Y \mid V\right)+I\left(X_{1} ; X_{2} \mid V\right), \\
& R_{0}+R_{1}+R_{2} \leq I\left(X_{1}, X_{2} ; Y\right)+I\left(X_{1} ; X_{2} \mid V\right), \\
& R_{0}+R_{1} \leq C_{1}, \\
& R_{0}+R_{2} \leq C_{2}, \\
& 0 \leq r_{1} \leq R_{1}, \\
& 0 \leq r_{2} \leq R_{2},
\end{aligned}
$$

for any $\delta>0$. We will show that rate $R$ defined as

$$
R=R_{0}+R_{1}-r_{1}+R_{2}-r_{2}
$$

is achievable.

Codebook generation: First randomly generate $2^{n R_{0}}$ many $v^{n}$ sequences according to $p(v)$ and index them as $v^{n}(1), \ldots, v^{n}\left(2^{n R_{0}}\right)$. The $v^{n}$ sequences constitute the inner codebook.

Conditioned on $v^{n}(i), i=1, \ldots, 2^{n R_{0}}$, for each $j=1,2, \ldots, 2^{n\left(R_{1}-r_{1}\right)}$, generate a subcodebook $C_{1}(i, j)$ consisting of $2^{n r_{1}}$ many $x_{1}^{n}$ sequence in a conditionally i.i.d. fashion according to $p\left(x_{1} \mid v\right)$. We index the codeword sequences in the subcodebook $C_{1}(i, j)$ as $x_{1}^{n}\left(i, l_{1}\right)$ for $l_{1}=(j-1) 2^{n r_{1}}+$ $1, \ldots, j 2^{n r_{1}}$. Similarly, conditioned on $v^{n}(i), i=1, \ldots, 2^{n R_{0}}$, for each $k=1,2, \ldots, 2^{n\left(R_{2}-r_{2}\right)}$, generate a subcodebook $C_{2}(i, k)$ consisting of $2^{n r_{2}}$ many $x_{2}^{n}$ sequences in a conditionally i.i.d. fashion according to $p\left(x_{2} \mid v\right)$. We index the codeword sequences in the subcodebook $C_{2}(i, k)$ as $x_{2}^{n}\left(i, l_{2}\right)$ for $l_{2}=(k-1) 2^{n r_{2}}+1, \ldots, k 2^{n r_{2}}$.

For each subcodebook pair $\left(C_{1}(i, j), C_{2}(i, k)\right), i=1, \ldots, 2^{n R_{0}}, j=1,2, \ldots, 2^{n\left(R_{1}-r_{1}\right)}$ and $k=1,2, \ldots, 2^{n\left(R_{2}-r_{2}\right)}$, find a pair of codewords $\left(x_{1}^{n}, x_{2}^{n}\right)$ such that $x_{1}^{n} \in C_{1}(i, j), x_{2}^{n} \in C_{2}(i, k)$ and $\left(x_{1}^{n}, x_{2}^{n}\right) \in \mathcal{T}_{\left[X_{1} X_{2} \mid V\right]}^{n}\left(v^{n}(i)\right)$, where $\mathcal{T}_{\left[X_{1} X_{2} \mid V\right]}^{n}\left(v^{n}(i)\right)$ is the conditional typical set according 


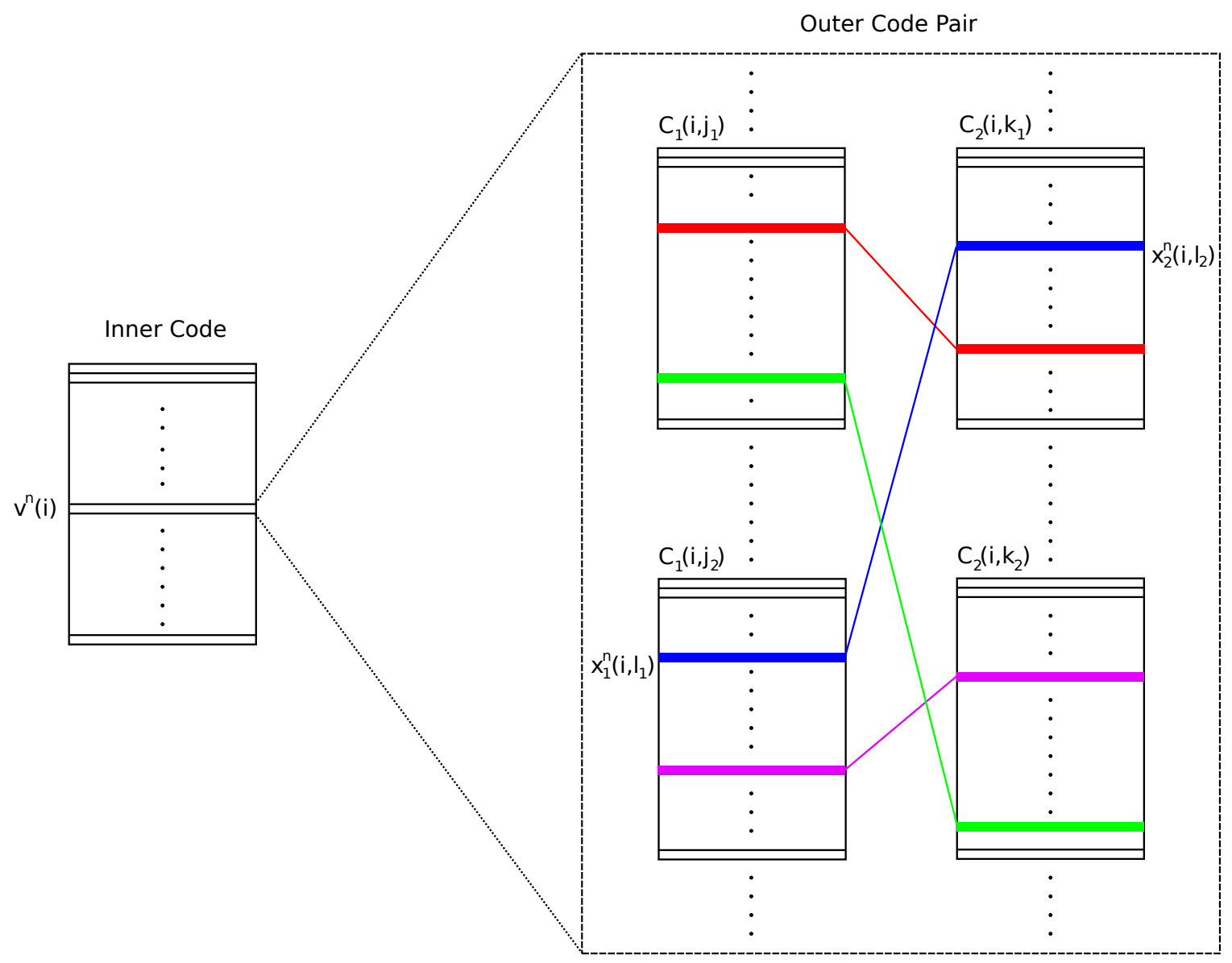

Fig. 6. The correlated codes with superposition structure.

to the distribution $p\left(x_{1}, x_{2} \mid v\right)$ [16]. If there are multiple such codeword pairs, then pick one pair randomly. If there is no such pair, we randomly choose a pair of $\left(x_{1}^{n}, x_{2}^{n}\right)$ from the subcodebook pair $\left(C_{1}(i, j), C_{2}(i, k)\right)$. We denote the picked codeword pair, say $\left(x_{1}^{n}\left(i, l_{1}\right), x_{2}^{n}\left(i, l_{2}\right)\right)$, as the $(i, j, k)$-th codeword pair. We also define the $i$-th correlated codebook $\mathcal{C}(i)$, which contains all the $(i, j, k)$-th codeword pair for $j=1,2, \ldots, 2^{n\left(R_{1}-r_{1}\right)}$ and $k=1,2, \ldots, 2^{n\left(R_{2}-r_{2}\right)}$. We illustrate the structure of inner code and the outer code graphically in Fig. 6 ,

Encoding: We split the message $W$, which is uniformly distributed on $\left\{1,2, \ldots, 2^{n R}\right\}$, into $\left(W_{a}, W_{b}, W_{c}\right)$, where $W_{a}, W_{b}$ and $W_{c}$ are uniformly distributed on $\left\{1,2, \ldots, 2^{n R_{0}}\right\}$ ,$\left\{1,2, \ldots, 2^{n\left(R_{1}-r_{1}\right)}\right\}$ and $\left\{1,2, \ldots, 2^{n\left(R_{2}-r_{2}\right)}\right\}$, respectively. When $\left(W_{a}, W_{b}, W_{c}\right)=(i, j, k)$, we select the $(i, j, k)$-th codeword pair from the subcodebook pair $\left(C_{1}(i, j), C_{2}(i, k)\right)$, i.e., $\left(x_{1}^{n}\left(i, l_{1}\right)\right.$, $\left.x_{2}^{n}\left(i, l_{2}\right)\right)$. The transmitter sends index $\left(i, l_{1}\right)$ to Relay 1 and index $\left(i, l_{2}\right)$ to Relay 2 . The relays 
can correctly receive the indices due to $R_{0}+R_{1} \leq C_{1}$ in (47) and $R_{0}+R_{2} \leq C_{2}$ in (48). Upon receiving the index $(i, l)$, Relay 1 sends the codeword $x_{1}^{n}\left(i, l_{1}\right)$ into the multiple access channel and similarly, upon receiving the index $\left(i, l_{2}\right)$, Relay 2 sends the codeword $x_{2}^{n}\left(i, l_{2}\right)$ into the multiple access channel.

Decoding: After receiving $y^{n}$, if there exists a unique codeword pair $\left(x_{1}^{n}\left(i, l_{1}\right), x_{2}^{n}\left(i, l_{2}\right)\right)$ which is the $(i, j, k)$-th codeword pair from the subcodebook pair $\left(C_{1}(i, j), C_{2}(i, k)\right)$, such that

$$
\left(v^{n}(i), x_{1}^{n}\left(i, l_{1}\right), x_{2}^{n}\left(i, l_{2}\right), y^{n}\right) \in \mathcal{T}_{\left[V X_{1} X_{2} Y\right]}^{n},
$$

where $\mathcal{T}_{\left[V X_{1} X_{2} Y\right]}^{n}$ is the typical set as defined in [16] according to $p\left(v, x_{1}, x_{2}, y\right)$, then the receiver declares $\left(W_{a}, W_{b}, W_{c}\right)=(i, j, k)$; otherwise, the receiver declares an error.

Probability of Error: Due to symmetry, the average probability of error is equivalent to the probability of error for an arbitrary message $w \in\left\{1, \ldots, 2^{n R}\right\}$. Hence, without loss of generality, we assume $W=w$, and the average probability of error satisfies

$$
\operatorname{Pr}[E] \triangleq \operatorname{Pr}\left[g^{n}\left(Y^{n}\right) \neq w \mid W=w\right], \quad w=1, \ldots, 2^{n R},
$$

For $W=w$, we denote $\left(W_{a}, W_{b}, W_{c}\right)=(i, j, k)$, and the corresponding codeword pair is denoted as $\left(x_{1}^{n}\left(i, l_{1}\right), x_{2}^{n}\left(i, l_{2}\right)\right)$. An error occurs if one of the following error events happen.

1) $E_{0}$ : there does not exist a pair of codewords $\left(x_{1}^{n}, x_{2}^{n}\right)$ such that $x_{1}^{n} \in C_{1}(i, j), x_{2}^{n} \in C_{2}(i, k)$ and $\left(x_{1}^{n}, x_{2}^{n}\right) \in \mathcal{T}_{\left[X_{1} X_{2} \mid V\right]}^{n}\left(v^{n}(i)\right)$.

2) $E_{1}:\left(v^{n}(i), x_{1}^{n}\left(i, l_{1}\right), x_{2}^{n}\left(i, l_{2}\right), Y^{n}\right) \notin \mathcal{T}_{\left[V X_{1} X_{2} Y\right]}^{n}$.

3) $E_{2}$ : There exist other codewords jointly typical with $Y^{n}$, which includes

a) $E_{21}$ : there exists $l_{1}^{\prime} \neq l_{1}$ such that $\left(x_{1}^{n}\left(i, l_{1}^{\prime}\right), x_{2}^{n}\left(i, l_{2}\right)\right) \in \mathcal{C}(i)$ and $\left(v^{n}(i), x_{1}^{n}\left(i, l_{1}^{\prime}\right)\right.$, $\left.x_{2}^{n}\left(i, l_{2}\right), Y^{n}\right) \in \mathcal{T}_{\left[V X_{1} X_{2} Y\right]}^{n}$,

b) $E_{22}$ : there exists $l_{2}^{\prime} \neq l_{2}$ such that $\left(x_{1}^{n}\left(i, l_{1}\right), x_{2}^{n}\left(i, l_{2}^{\prime}\right)\right) \in \mathcal{C}(i)$ and $\left(v^{n}(i), x_{1}^{n}(i, l)\right.$, $\left.x_{2}^{n}\left(i, l_{2}^{\prime}\right), Y^{n}\right) \in \mathcal{T}_{\left[V X_{1} X_{2} Y\right]}^{n}$;

c) $E_{23}$ : there exists $l_{1}^{\prime} \neq l_{1}, l_{2}^{\prime} \neq l_{2}$ such that $\left(x_{1}^{n}\left(i, l_{1}^{\prime}\right), x_{2}^{n}\left(i, l_{2}^{\prime}\right)\right) \in \mathcal{C}(i)$ and $\left(v^{n}(i)\right.$, $\left.x_{1}^{n}\left(i, l_{1}^{\prime}\right), x_{2}^{n}\left(i, l_{2}^{\prime}\right), Y^{n}\right) \in \mathcal{T}_{\left[V X_{1} X_{2} Y\right]}^{n} ;$

d) $E_{24}$ : there exists $i^{\prime} \neq i, l_{1}^{\prime}, l_{2}^{\prime}$ such that $\left(x_{1}^{n}\left(i^{\prime}, l_{1}^{\prime}\right), x_{2}^{n}\left(i^{\prime}, l_{2}^{\prime}\right)\right) \in \mathcal{C}\left(i^{\prime}\right)$ and $\left(v^{n}\left(i^{\prime}\right), x_{1}^{n}\left(i^{\prime}, l_{1}^{\prime}\right)\right.$, $\left.x_{2}^{n}\left(i^{\prime}, l_{2}^{\prime}\right), Y^{n}\right) \in \mathcal{T}_{\left[V X_{1} X_{2} Y\right]}^{n}$. 
Thus, the probability of error $\operatorname{Pr}[E]$ can be upper bounded as

$\operatorname{Pr}\left[E_{0}\right]+\operatorname{Pr}\left[E_{1} \mid E_{0}^{c}\right]+\operatorname{Pr}\left[E_{21} \mid E_{1}^{c} \cap E_{0}^{c}\right]+\operatorname{Pr}\left[E_{22} \mid E_{1}^{c} \cap E_{0}^{c}\right]+\operatorname{Pr}\left[E_{23} \mid E_{1}^{c} \cap E_{0}^{c}\right]+\operatorname{Pr}\left[E_{24} \mid E_{1}^{c} \cap E_{0}^{c}\right]$

We start with upper bounding $\operatorname{Pr}\left[E_{0}\right]$. From the mutual covering lemma [17], we have that $\operatorname{Pr}\left[E_{0}\right] \leq \epsilon$ if $r_{1}+r_{2}>I\left(X_{1} ; X_{2} \mid V\right)$. Therefore, if the inequality in (42) is satisfied, we have $\operatorname{Pr}\left[E_{0}\right] \leq \epsilon$

Due to the Asymptotic Equipartition Property (AEP), for sufficient large $n$, we have

$$
\operatorname{Pr}\left[E_{1} \mid E_{0}^{c}\right] \leq \epsilon
$$

Next, we proceed to upper bound $\operatorname{Pr}\left[E_{21} \mid E_{1}^{c} \cap E_{0}^{c}\right]$. We have

$$
\begin{aligned}
\operatorname{Pr}\left[E_{21} \mid E_{0}^{c} \cap E_{1}^{c}\right] & \leq \sum_{l_{1}^{\prime} \neq l_{1}: x_{1}^{n}\left(i, l_{1}^{\prime}\right)} \operatorname{Pr}\left[\left(v^{n}(i), x_{1}^{n}\left(i, l_{1}^{\prime}\right), x_{2}^{n}\left(i, l_{2}\right), Y^{n}\right) \in \mathcal{T}_{\left[V X_{1} X_{2} Y\right]}^{n} \mid E_{1}^{c} \cap E_{0}^{c},\right] \\
& \leq 2^{-n\left(I\left(X_{1} ; Y, X_{2} \mid V\right)-\epsilon\right)} 2^{n R_{1}} .
\end{aligned}
$$

which goes to zero because of (43). Due to symmetry, the probability $\operatorname{Pr}\left[E_{22} \mid E_{1}^{c} \cap E_{0}^{c}\right]$ goes to zero because of (44). The probability $\operatorname{Pr}\left[E_{23} \mid E_{1}^{c} \cap E_{2}^{c}\right]$ can be bounded as follows

$$
\begin{aligned}
& \operatorname{Pr}\left[E_{23} \mid E_{1}^{c} \cap E_{0}^{c}\right] \\
& =\sum_{l_{1}^{\prime} \neq l_{1}, l_{2}^{\prime} \neq l_{2},\left(x_{1}^{n}\left(i, l_{1}^{\prime}\right), x_{2}^{n}\left(i, l_{2}^{\prime}\right)\right) \in \mathcal{C}(i)} \operatorname{Pr}\left[\left(v^{n}(i), x_{1}^{n}\left(i, l_{1}^{\prime}\right), x_{2}^{n}\left(i, l_{2}^{\prime}\right), Y^{n}\right) \in \mathcal{T}_{\left[V X_{1} X_{2} Y\right]}^{n} \mid E_{1}^{c} \cap E_{0}^{c}\right] \\
& \leq 2^{-n\left(I\left(X_{1}, X_{2} ; Y \mid V\right)-\epsilon\right)} 2^{n\left(R_{1}-r_{1}+R_{2}-r_{2}\right)} \\
& \leq 2^{-n\left(I\left(X_{1}, X_{2} ; Y \mid V\right)-\epsilon\right)} 2^{n\left(R_{1}+R_{2}-I\left(X_{1} ; X_{2} \mid V\right)-\delta\right)} .
\end{aligned}
$$

where the inequality in (54) is due to (42) and the quantities in (54) goes to zero due to (45). The probability $\operatorname{Pr}\left[E_{24} \mid E_{1}^{c} \cap E_{2}^{c}\right]$ can be bounded as follows

$$
\begin{aligned}
& \operatorname{Pr}\left[E_{24} \mid E_{1}^{c} \cap E_{0}^{c}\right] \\
& =\sum_{i^{\prime} \neq i, l_{1}^{\prime}, l_{2}^{\prime}\left(x_{1}^{n}\left(i^{\prime}, l_{1}^{\prime}\right), x_{2}^{n}\left(i^{\prime}, l_{2}^{\prime}\right)\right) \in \mathcal{C}\left(i^{\prime}\right)} \operatorname{Pr}\left[\left(v^{n}\left(i^{\prime}\right), x_{1}^{n}\left(i^{\prime}, l_{1}^{\prime}\right), x_{2}^{n}\left(i^{\prime}, l_{2}^{\prime}\right), Y^{n}\right) \in \mathcal{T}_{\left[V X_{1} X_{2} Y\right]}^{n} \mid E_{1}^{c} \cap E_{0}^{c}\right] \\
& \leq 2^{-n\left(I\left(X_{1}, X_{2} ; Y\right)-\epsilon\right)} 2^{n R_{0}} 2^{n\left(R_{1}-r_{1}+R_{2}-r_{2}\right)} \\
& \leq 2^{-n\left(I\left(X_{1}, X_{2} ; Y\right)-\epsilon\right)} 2^{n\left(R_{0}+R_{1}+R_{2}-I\left(X_{1} ; X_{2} \mid V\right)-\delta\right)}
\end{aligned}
$$




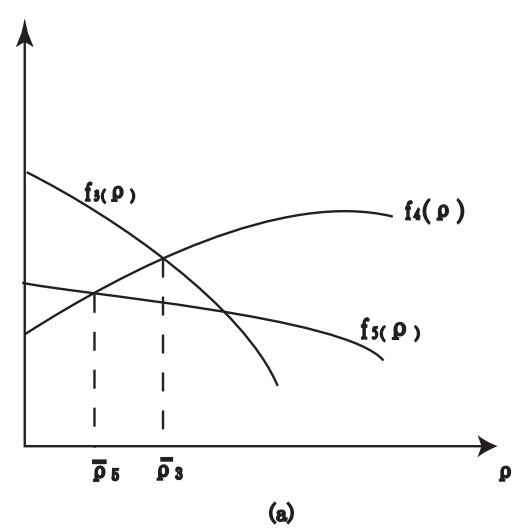

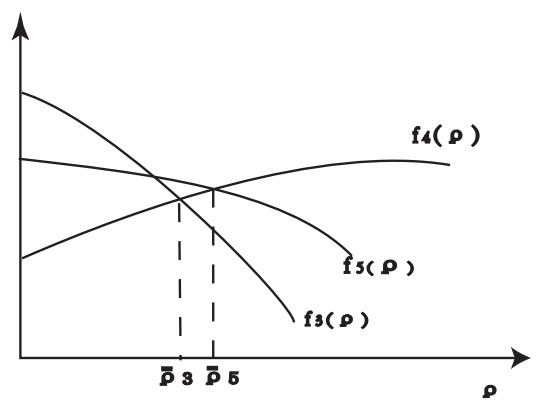

(b)

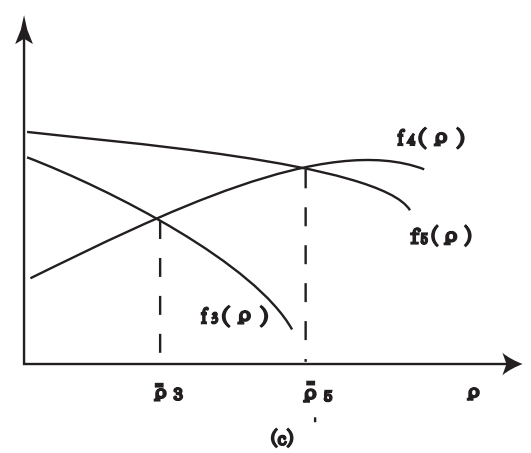

Fig. 7. The possible cases for $f_{3}, f_{4}$ and $f_{5}$.

which goes to zero due to (46). Thus, as long as the rate tuple satisfies (42)-(50), the probability of error can be made arbitrarily small, and rate $R$ according to (51) is achievable. Using FourierMotzkin elimination and noting the fact that the capacity is defined as the supremum of all achievable rates, we obtain the result stated in Theorem 2 .

\section{Proof of Theorem 3}

We first show that if the Gaussian multiple access diamond channel satisfies (7) and (8), then $\bar{\rho}_{3}$ and $\bar{\rho}_{5}$ always exist. Since the channel satisfies ([8), we have $f_{3}(0)>f_{4}(0)$ and $f_{5}(0)>f_{4}(0)$. Since the channel satisfies (7), we have $f_{5}(1)<f_{4}(1)$. Furthermore, we always have $f_{3}(1)<$ $f_{4}(1)$. Since all functions $f_{3}, f_{4}$ and $f_{5}$ are continuous, by the intermediate value theorem, $\bar{\rho}_{3}$ and $\bar{\rho}_{5}$ always exist. Furthermore, since $f_{3}(\rho), f_{4}(\rho)$ and $f_{5}(\rho)$ are all monotonic, the root $\bar{\rho}_{k} \in[0,1]$, $k=3,5$ is unique.

It is clear that $f_{3}(\rho)$ and $f_{5}(\rho)$ are strictly decreasing in $\rho$, while $f_{4}(\rho)$ is strictly increasing in $\rho$, for $\rho \in[0,1]$. We also know that $f_{3}(1)<f_{5}(1)$. Noting that both $f_{3}(\rho)$ and $f_{5}(\rho)$ are functions of $\rho$ in terms of $1-\rho^{2}$ only, it can be shown that $f_{3}(\rho)=f_{5}(\rho)$ have at most one root in $\rho \in[0,1]$. Thus, if the channel further satisfies $f_{3}(0)>f_{5}(0)$, then $f_{3}, f_{4}$ and $f_{5}$ would look like either Figure 7 (a) or Figure 7 (b). Otherwise, $f_{3}, f_{4}$ and $f_{5}$ would look like Figure 7 (c).

We discuss the following cases:

1) In the case of Figure $7(\mathrm{a}), f_{3}(0)>f_{5}(0)$ and $\bar{\rho}_{5} \leq \bar{\rho}_{3}$. In this scenario, the lower bound is $f_{4}\left(\bar{\rho}_{5}\right)$. The cut-set bound is also $f_{4}\left(\bar{\rho}_{5}\right)$. Thus, in this case, we have $C_{\text {lower }}=C_{\text {upper }}=C_{\text {cut }}$. 
2) In the case of Figure $7(\mathrm{~b}), f_{3}(0)>f_{5}(0)$ and $\bar{\rho}_{3}<\bar{\rho}_{5}$. In this scenario, the lower bound is $f_{4}\left(\bar{\rho}_{3}\right)$. The cut-set bound is $f_{4}\left(\bar{\rho}_{5}\right)$. As for the upper bound, we have the following sub-cases:

a) if $\rho^{*} \leq \bar{\rho}_{3}$, then $T_{1}=f_{4}\left(\rho^{*}\right)$ and $T_{2}=f_{4}\left(\bar{\rho}_{5}\right)$. Since $T_{1}<T_{2}$, the upper bound is $f_{4}\left(\bar{\rho}_{5}\right)$. Thus, in this case, we have $C_{\text {lower }}<C_{\text {upper }}=C_{\text {cut }}$.

b) If $\bar{\rho}_{5} \geq \rho^{*}>\bar{\rho}_{3}$, then $T_{1}=f_{4}\left(\bar{\rho}_{3}\right), T_{2}=f_{4}\left(\bar{\rho}_{5}\right)$ and $T_{1}<T_{2}$, the upper bound is $f_{4}\left(\bar{\rho}_{5}\right)$. Thus, in this case, we have $C_{\text {lower }}<C_{\text {upper }}=C_{\text {cut }}$.

c) If $\rho^{*}>\bar{\rho}_{5}$, then $T_{1}=f_{4}\left(\bar{\rho}_{3}\right), T_{2}=f_{5}\left(\rho^{*}\right)$ and the upper bound is $\max \left(f_{4}\left(\bar{\rho}_{3}\right), f_{5}\left(\rho^{*}\right)\right)$.

Thus, we have the following 2 sub-cases:

i) $f_{5}\left(\rho^{*}\right) \leq f_{4}\left(\bar{\rho}_{3}\right)$. In this case, we have we have $C_{\text {lower }}=C_{\text {upper }}<C_{\text {cut }}$.

ii) $f_{5}\left(\rho^{*}\right)>f_{4}\left(\bar{\rho}_{3}\right)$. In this case, we have $C_{\text {lower }}<C_{\text {upper }}<C_{\text {cut }}$.

3) In the case of Figure 7 (c), $f_{3}(0) \leq f_{5}(0)$. Since we have $f_{3}(1)<f_{5}(1)$, we have that $\bar{\rho}_{3}<\bar{\rho}_{5}$ in this case. The lower bound is $f_{4}\left(\bar{\rho}_{3}\right)$. The cut-set bound is $\min \left(f_{3}(0), f_{4}\left(\bar{\rho}_{5}\right)\right)$. As for the upper bound, we have the following sub-cases:

a) if $\rho^{*} \leq \bar{\rho}_{3}$, then $T_{1}=f_{4}\left(\rho^{*}\right)$ and $T_{2}=\min \left(f_{3}(0), f_{4}\left(\bar{\rho}_{5}\right)\right)$. Since $T_{1}<T_{2}$, the upper bound is $\min \left(f_{3}(0), f_{4}\left(\bar{\rho}_{5}\right)\right)$. Thus, in this case, we have $C_{\text {lower }}<C_{\text {upper }}=C_{\text {cut }}$.

b) If $\bar{\rho}_{3}<\rho^{*} \leq \bar{\rho}_{5}$, then $T_{1}=f_{4}\left(\bar{\rho}_{3}\right), T_{2}=\min \left(f_{3}(0), f_{4}\left(\bar{\rho}_{5}\right)\right)$. Note that $f_{4}\left(\bar{\rho}_{3}\right)=$ $f_{3}\left(\bar{\rho}_{3}\right)<f_{3}(0)$. Thus, $T_{1}<T_{2}$, the upper bound is $\min \left(f_{3}(0), f_{4}\left(\bar{\rho}_{5}\right)\right)$. Thus, in this case, we have $C_{\text {lower }}<C_{\text {upper }}=C_{\text {cut }}$.

c) If $\rho^{*}>\bar{\rho}_{5}$, then $T_{1}=f_{4}\left(\bar{\rho}_{3}\right), T_{2}=\min \left(f_{3}(0), f_{5}\left(\rho^{*}\right)\right)$ and the upper bound is $\max \left(f_{4}\left(\bar{\rho}_{3}\right), \min \left(f_{5}\left(\rho^{*}\right), f_{3}(0)\right)\right)$. we further have the following sub-cases:

i) $f_{5}\left(\rho^{*}\right) \leq f_{4}\left(\bar{\rho}_{3}\right)$, we have $C_{\text {lower }}=C_{\text {upper }}<C_{\text {cut }}$.

ii) $f_{5}\left(\rho^{*}\right)>f_{4}\left(\bar{\rho}_{3}\right)$, then the upper bound is $\min \left(f_{5}\left(\rho^{*}\right), f_{3}(0)\right)$, we further have the following cases:

A) $f_{3}(0) \leq f_{5}\left(\rho^{*}\right)$. In this case, $C_{\text {upper }}=f_{3}(0)$. Since we are considering the case of $\rho^{*}>\bar{\rho}_{5}$, and due to the fact that $f_{5}(\cdot)$ is a decreasing function, we have $C_{\text {cut }}=f_{3}(0)$. Thus, in this case, we have $C_{\text {lower }}<C_{\text {upper }}=C_{\text {cut }}$.

B) $f_{3}(0)>f_{5}\left(\rho^{*}\right)$. In this case, $C_{\text {upper }}=f_{5}\left(\rho^{*}\right)$, and we have $C_{\text {lower }}<C_{\text {upper }}<$ $C_{\text {cut }}$.

Combining the result for these cases, and noting that we have $\bar{\rho}_{5} \leq \bar{\rho}_{3}$ implies $f_{3}(0)>f_{5}(0)$, 
$f_{5}\left(\rho^{*}\right) \leq f_{4}\left(\bar{\rho}_{3}\right)$, together with $\bar{\rho}_{3}<\bar{\rho}_{5}$, implies $\rho^{*}>\bar{\rho}_{5}, f_{3}(0)>f_{5}(0)$ implies $f_{3}(0)>f_{5}\left(\rho^{*}\right)$, $f_{5}\left(\rho^{*}\right) \leq f_{4}\left(\bar{\rho}_{3}\right)$ implies $f_{5}\left(\rho^{*}\right)<f_{3}(0)$, we obtain the result of Theorem 3 ,

\section{Conclusions}

We have studied the Gaussian multiple access diamond channel. Noting the similarity between this problem and the Gaussian multiple description problem, we first provide an upper bound on the capacity. We then obtain an achievable rate by correlated code with the superposition structure. Finally, we provide conditions that our proposed upper and lower bounds meet. Thus, for a Gaussian multiple access diamond channel that satisfies these conditions, we have found its capacity.

\section{REFERENCES}

[1] B. E. Schein. Distributed Coordination in Network Information Theory. PhD thesis, Massachusetts Institute of Technology, 2001.

[2] T. M. Cover and J. A. Thomas. Elements of Information Theory. John Wiley and Sons, 1991.

[3] W. Kang and S. Ulukus. Capacity of a class of diamond channels. IEEE Trans. Inform. Theory, 57:4955-4960, Aug. 2011.

[4] R. Tandon and S. Ulukus. Diamond channel with partially separated relays. In Proc. IEEE International Symp. on Information Theory (ISIT), pages 644-648, Austin, TX, June 2010.

[5] A. S. Avestimehr, S. N. Diggavi, and D. N. C. Tse. Wireless network information flow: A deterministic approach. IEEE Trans. Inform. Theory, 57(4):1872-1905, Apr. 2011.

[6] U. Niesen and S. N. Diggavi. The approximate capacity of the Gaussian N-relay diamond network. IEEE Trans. Inform. Theory, 59(2):845-859, Feb. 2013.

[7] R. Ahlswede and T. S. Han. On source coding with side information via a multiple-access channel and related problems in multi-user information theory. IEEE Trans. Inform. Theory, 29(3):396-412, 1983.

[8] D. Traskov and G. Kramer. Reliable communication in networks with multi-access interference. In Proc. Conf. IEEE Information Theory Workshop (ITW), Lake Tahoe, CA, Sep. 2007.

[9] T. M. Cover, A. El Gamal, and M. Salehi. Multiple access channel with arbitrarily correlated sources. IEEE Trans. Inform. Theory, 26:648-657, Nov. 1980.

[10] F. M. J. Willems. The discrete memoryless multiple access channel with partially cooperating encoders. IEEE Trans. Inform. Theory, 29(3):441-445, May 1983.

[11] S. I. Bross, A. Lapidoth, and M. A. Wigger. The Gaussian MAC with conferencing encoders. In Information Theory, 2008. ISIT 2008. IEEE International Symposium on, pages 2702-2706, 2008.

[12] K. Marton. A coding theorem for the discrete memoryless broadcast channel. IEEE Trans. Inform. Theory, 25:306-311, May 1979.

[13] L. Ozarow. On a source-coding problem with two channels and three receivers. Bell Syst. Tech. J., 59:1909-1921, December 1980 . 
[14] P. Bergmans. A simple converse for broadcast channels with additive white Gaussian noise. IEEE Trans. on Information Theory, 20:279 -280, March 1974.

[15] S. S. Bidokhti and G. Kramer. Capacity bounds for a class of diamond networks. arXiv preprint arXiv:1401.6135, 2014.

[16] I. Csiszar and J. Korner. Information Theory: Coding Theorems for Discrete Memoryless Systems. Academic Press, 1981.

[17] A. El Gamal and Y. H. Kim. Network information theory. Cambridge University Press, 2011. 